\title{
Formation of ultra-thin bi-molecular boundary adsorbed films
}

\author{
W. W. F. Chong ${ }^{1}$, M. Teodorescu ${ }^{1,2}$ and H. Rahnejat ${ }^{3}$ \\ ${ }^{1}$ School of Engineering, Cranfield University, Cranfield, UK \\ 2 Baskin School of Engineering, University of California at Santa Cruz, CA USA \\ ${ }^{3}$ Wolfson School of Mechanical \& Manufacturing Engineering, Loughborough \\ University, Loughborough, UK \\ E-mail: mteodorescu@soe.ucsc.edu
}

\begin{abstract}
.
An analytical method based on statistical mechanics is proposed for the prediction of ultra-thin adsorbed films of physical fluids of molecular diversity formed on smooth surfaces. The model is representative of molecular interactions at the smooth summits of surface asperities in the nano-scale. At this physical scale the constraining effect of the solid barriers promotes discretisation of the fluid volume into molecular layers, which are usually ejected from the contact in a stepwise manner. The integrated effect of intermolecular interactions of molecular species as well as their interactions with the contiguous surfaces is responsible for this discontinuous drainage of the fluid. However, at the same time, the adsorption energy of the molecular species strives to form a molecular mono-layer upon the boundary solids. The net result of these complex interactions is an ultra-thin adsorbed film whose shear characteristics depends on a competition between the repulsive solvation pressure and the energy of molecular adsorption. It is shown that very thin low shear strength films formed in this manner depend on ideal molecular concentration and wall adsorption energy. An important implication is that boundary adherent films should be viewed as a result of surfacefluid combination for which choice of concentration and fraction content of particular species are crucial.
\end{abstract}

Keyword: Statistical mechanics, Boundary films, Solvation, adsorption 


\section{Introduction}

Formation of ultra-thin surface adsorbed films is crucial in smooth running of many conjunctions in variety of applications. However, unlike the well-understood lubricant films formed by hydrodynamic viscous action, the knowledge regarding the mechanisms underlying surface or boundary films is still emerging. These thin surface films have low shear strength characteristics and are formed as the result of tribo-chemical interactions between molecules of the lubricant and the surface material. Therefore, the study of lubricant-surface interaction is critical for their understanding and their role in friction. In fact, a recent review by Erdemir [1] shows that lubricant-surface combination must be considered as an integrated system. The review shows that there has been a significant growing volume of research on tribo-films. However, formation of these films is an effect rather than the underlying physio-chemical cause which is due to the intermolecular interactions of a fluid species, as well as between the molecules with the atoms of the bounding solid surfaces. It is, therefore, necessary to understand the plethora of kinetic laws that belie these interactions. The multitude of molecular species and the seemingly abundant kinetic laws in the nano-scale promote a slow progress in understanding.

Molecules do not move in a straight line path, but in step-wise fashion and in all directions. Einstein and Infeld [2] showed that the mean squared distance moved by assumed hard spherical molecules in Brownian motion is linearly proportional to the elapsed time (or the rate of shear). Thus, surface adsorbed or boundary films may be considered to act as classical fluids with hard particles in a definite volume [3]. Real fluids, though, cannot be considered as idealized classical fluids. The boundary solids are also rough and often anisotropic. Thus, in the diminishing scale, the interaction potential between species of molecules and atoms of surfaces vary.

The complexity of the problem is exacerbated in lightly loaded minute contacts, particularly with very smooth surfaces, such as asperity summits. There are repercussions for emerging technologies such as micro-electromechanical systems and some data storage devices. The same is also true in contacts of low load intensity due to closer contiguity of bounding surfaces. Owing to free surface energy and intermolecular interactions the packing order of the molecules in such conjunctions alters near the solid barriers. Often any film of fluid exhibits a hierarchical nature according to the interaction scale. At diminutive separations the intermolecular interactions comprise long range van der Waals interactions between the fluid molecular species, as well as with the atoms of the solid barriers [4]. For charged surfaces and/or polar fluids these can be augmented by electrostatic interactions [5]. Additionally, any formed film of fluid has a different packing of molecules, several molecules deep into its bulk. Due to the free surface energy the fluid molecular density is higher at the solid barriers than in the bulk [6]. With increased loading or convergence of the bounding surfaces an oscillatory monotonic-exponential force results, known as the solvation force [6, 7]. Horn and Israelachvili [8] and Chan and Horn [9] have measured this oscillatory force between very smooth mica surfaces in liquids such as Octamethyltetracyclosiloxane (OMCTS), 
Hexadecane and Tetradecane, using surface force apparatus (SFA). Lim and O'Shea [10] used an atomic force microscope (AFM) to measure the solvation force for fluid mixtures.

Various theoretical models have been developed, where conjunctional behaviour is an amalgam of a mix of kinetic laws: from hydrodynamics to surface energy effects, as well as intermolecular interactions. They include the works reported by Matsuoka and Kato [11], Al-Samieh and Rahnejat [12, 13, 14] and Jang and Tichy [15] in the study of lightly loaded conjunctions with diminutively thin films, entrained through by hydrodynamic viscous action. To describe the solvation force, they have all adopted the use of Ornstein-Zernike (OZ) equation as expounded by Mitchel et al [16], Henderson and Lozada-Cassou [17] and Attard and Parker [7]. The OZ equation is based on the Percus-Yevick (PY) [18] approximation to predict the pair correlation function; PCF, $g^{*}$ between hard spherical molecules. The solvation force can then be predicted by applying the Derjaguin approximation for the interaction between usually flat planar surfaces. Alternatively, Monte-Carlo simulation and density functional theory can be used to predict the solvation force as in the case of works of Snook and Van Megen [19], Tarazona and Vicente [20], Somers et al [21] and Karanikas et al [22].

Matsuoka and Kato [11] and Al-Samieh and Rahnejat [12, 13] also include the effect of long range van der Waals forces, the latter using the classical Lifshitz theory [4]. They showed that with regard to surface adsorbed films of several molecular diameter of a basic mono-molecular non-polar fluid the effect of van der Waals interactions is quite insignificant. Later, Al-Samieh and Rahnejat [14] extended the kinetic laws in ultra-thin film conjunctions to include the squeeze film hydrodynamic action as well as meniscus effect in the case of lightly loaded impacts. They showed that mutual approach of smooth surfaces inhibits solvation by increasing the load capacity of the thin surface films. This was also confirmed by Teodorescu et al [23] in a subsequent study. Al-Samieh and Rahnejat [14] also included the effect of meniscus force in the vanishing conjunction of a pair of very smooth bounding solids. They showed that for an ideal fluid of mono-molecular species of assumed hard spherical form, the meniscus action constitutes the limit of oscillatory solvation behaviour for films of two molecules in depth. In fact, Chan and Horn's initial experiment which led to the observation of discrete molecular drainage from a lightly loaded contact made provisions to counteract the effect of meniscus action.

The empirical approach of Chan and Horn [9], however, is rather limited to a few types of basic fluids such as OMCTS. Most lubricants comprise a mix of molecular species, many of which are long hydrocarbon chains, esters and surfactants whose interactions do not conform to those of assumed basic hard impenetrable spheres, which mimic short range characteristics of atoms at close range [3]. Therefore, for real lubricants one is compelled to use molecular dynamics, Monte-Carlo simulations or statistical mechanics. These approaches are computationally time intensive and whilst, they may suit studies of regional colloidal behaviour, they are not suited to predict boundary adsorbed films in contacts of any significant dimensions. Surface adsorbed 
films act at the assumed smooth summits of surface features which are ubiquitous in any micro-scale contact according to some statistical distribution. If an analytical model for such a typical feature can be derived, then it may be included in an assumed statistical treatment of surface interactions in much the same manner as adhesion of asperities noted by Fuller and Tabor [24] and Johnson and Sridhar [25]. In fact, a combination of kinetic laws may be included in such an analysis as shown for adhesion, meniscus action and hydration by Rahnejat et al [26] and Teodorescu et al [23] for nano-scale lubricated impact dynamics of a diminutive roller and those of microelectromechanical systems (MEMS) gear teeth pairs respectively.

When a film of fluid is entrained into a conjunction through hydrodynamic action, the classical OZ theory may be used to account for the intermolecular and surface forces of classical fluids of hard spherical molecules as functions of molecular diameter and intermolecular spacing or distance from a solid barrier. In a recent paper, Chong et al [27] showed that near surface effects dominate in gaps of the order of several molecular diameters. They also showed that spherical molecules tend to solvate near assumed smooth surfaces of asperities at nano-scale. Their discrete drainage at steadily decreasing gaps adds to friction of any bulk lubricant film. However, at the same time this action generates solvation pressures which increase the load carrying capacity. Conversely, long chain molecules of smaller effective diameter tend to inhibit solvation, thus show a decrease in the load carrying capacity whilst through their wetting action reduce friction. The study also included the effect of thin film elastohydrodynamics with inlet lubricant starvation and outlet cavitation and film reformation boundaries.

In an evolutionary process to extend the work of Chong et al [27], it is necessary to develop a realistic physio-chemical hydrodynamic model, where intermolecular and surface interactions account for a mix of lubricant molecular species. The current contribution supplements the solution of OZ equation with an attractive narrow well potential to describe the characteristics of a simplified real physical fluid system through PY approximation. The solid planar boundary is also considered as a species in the analysis, where the interactions of hard spheres with its atoms are also taken into account. In the solution of the OZ equation with PY approximation, adhesion energy of hard spherical molecular species with the solid planar boundaries are allowed. Thus, the work of Chong et al [27] is not only extended from an idealized fluid to a physical one, but also includes the adsorption/adhesion of molecules to the solid boundaries. This is the prelude to the formation of tribo-films of interest in boundary lubrication. A basic lubricant is assumed with spherical molecules. This is supplemented with different concentrations of an additive, represented by a smaller diameter spherical molecule with different levels of adsorption energy. In reality, the additive molecules are long chain structures, but with a slender cross-section. These often adsorb to the surfaces through their small diameter terminus with their backbone comprising a long chain tentacle. 


\section{Mathematical Model}

The fluid between two surfaces can be modelled as a large number of interacting molecules, which in the first approximation can be viewed as hard spheres $[7,11,28]$. These are defined as impenetrable particles of diameter, $\sigma_{\alpha}$ and $\sigma_{\beta}$ at a core to core distance of $r^{*}$ (see inset figure 1). The solvation pressure between approaching surfaces arises as a result of the fluid particles being constrained in a narrow gap with the same order of magnitude as their molecular size.

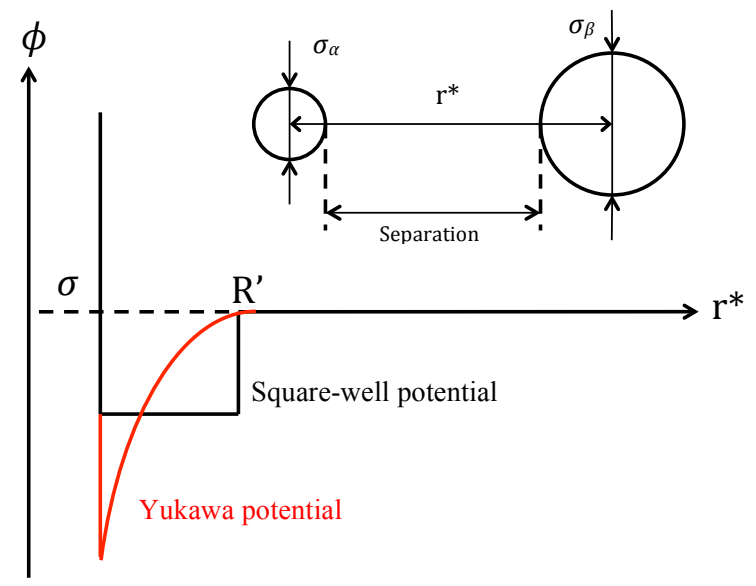

Figure 1. Particle interaction potentials (e.g. square-well and Yukawa potential)

\subsection{Solvation pressure}

Derjaguin describes the interplay of the hard spheres confined by macroscopic flat bodies using the interaction energy, $W^{*}[6,29]$, approximated by

$$
W^{*}\left(r^{*}\right)=\frac{1}{2 \pi R_{e f f}} \cdot \frac{d}{d r^{*}}\left[k_{B} T\left(g^{*}\left(r^{*}\right)-1\right)\right]
$$

where $R_{e f f}=\sigma_{\alpha} \sigma_{\beta} / 2\left(\sigma_{\alpha}+\sigma_{\beta}\right)$ is the effective diameter, $k_{B}$ is the Boltzmann constant, $T$ is the temperature of the fluid system, $\alpha$ and $\beta$ refer to the hard sphere species and $g^{*}\left(r^{*}\right)$ is the pair correlation function at distance $r^{*}$ (which is described later).

The solvation pressure, $p_{s}$ generated by hard spheres, confined by flat bodies can be obtained from equation (1):

$$
p_{s}=-\frac{d}{d r^{*}} W^{*}\left(r^{*}\right)
$$

The pair correlation function $\left(g_{\alpha \beta}^{*}\right.$ in equation (1)) describes the probability of finding a particle of species $\beta$ at a core to core distance, $r^{*}$ from a referenced particle: species $\alpha$. For a uniform fluid consisting of particles interacting through central forces, the pair correlation function, $g_{\alpha \beta}^{*}$ is expressed as: 


$$
g_{\alpha \beta}^{*}\left(r^{*}\right)=h_{\alpha \beta}^{*}\left(r^{*}\right)+1, \quad \alpha, \beta \in\{1,2,3 \ldots(M+1)\}
$$

where $h_{\alpha \beta}^{*}$ is the indirect correlation function, which describes the indirect influence of a particle of species $\alpha$ towards another particle of species $\beta$ through an intervening particle, which can be either of species $\alpha$ or $\beta$.

Ornstein and Zernike [30] proposed the following equation (OZ equation) for the the indirect correlation function $\left(h_{\alpha \beta}^{*}\right)$ :

$$
h_{\alpha \beta}^{*}\left(r^{*}\right)=c_{\alpha \beta}^{*}+\sum_{\gamma=1}^{M+1} \rho_{\gamma}^{*} \int c_{\alpha \gamma}^{*}(|s|) h_{\gamma \beta}^{*}\left(\left|r^{*}-s\right|\right) d s
$$

where $(M+1)$ is the total number of hard sphere species and $s$ is the core to core distance of the surrounding spherical particles in a fluidic system towards a reference particle, either of species $\alpha$ or $\beta$. The direct correlation function, $c_{\alpha \beta}^{*}$ determines the interaction between the spherical particles of species $\alpha$ and $\beta$. The parameter $\rho_{\gamma}^{*}$ refers to the number density or particle density for the hard spheres of species $\gamma \in\{\alpha, \beta\}$.

Solving the OZ equation requires a suitable expression for the direct correlation function. The most effective ones are [7, 31, 32]:

1) Percus-Yevick (PY) approximation

$$
c_{\alpha \beta}^{*}\left(r^{*}\right)=g_{\alpha \beta}^{*} \cdot\left(r^{*}\right)\left[1-e^{\phi_{\alpha \beta}\left(r^{*}\right) / k_{B} T}\right]
$$

2) Convolution-Hypernetted Chain (CHNC)

$$
c_{\alpha \beta}^{*}\left(r^{*}\right)=h_{\alpha \beta}^{*}\left(r^{*}\right)-\log g_{\alpha \beta}^{*}\left(r^{*}\right)-\phi_{\alpha \beta}\left(r^{*}\right) /\left(k_{B} T\right)
$$

The attractive potential $\phi_{\alpha \beta}$ describes the adsorption between molecules $\alpha$ and $\beta$ [31]. The adsorption refers to the adhesion of particles, which form a molecularly-thin layer adhered to the surface. The PY approximation is preferred in this study because CHNC often causes divergence in numerical solutions of multi-molecule fluids [33]. Additionally, PY approximation is easier to apply and for strongly repulsive interactions (e.g. solvation) leads to accurate results [34].

Low concentrations of friction modifiers are often added to the base oil to decrease the boundary friction. These adhere to the surfaces, providing a protective layer with minimal shear resistance [35]. Friction modifiers are long chain molecules, typically alkane molecules with ten or more carbon atoms and a complex surface-active group at their ends [36].

Jahanmir and Beltzer [37] provide an empirical model for the boundary friction coefficient using a two-component lubricant. They used Temkin as a base isothermal adsorption model [38] and assumed that friction arises only due to direct surface 
interaction. This approach accounts for inhomogeneity in the contacting surfaces, but it is limited to the weakly adsorbed base oil. The conclusion was that increasing lubricant adsorption energy reduces the friction coefficient.

Baxter [28] describes the adsorption of molecules to surfaces based on infinitesimally short range potentials and solves the Ornstein-Zernike equation analytically using the Percus-Yevick approximation (OZ-PY). The adsorbent-adsorbate attraction is characterised by a single energy parameter. Therefore, the spheres coming into direct contact with the adsorbate planar surface are deemed to be adsorbed. Dickinson [39] extended the multi-component sticky hard spheres' model to a qualitative model, which could explain the competitive adsorption of protein molecules.

Figure 1 shows the most commonly used attractive potentials. These are the squarewell attraction and the Yukawa potential. Due to its simplicity and versatility, the square-well attraction potential is usually preferred (see figure 1) [40]. The infinitely narrow attractive well potential, $\phi$ for a single component fluid can be expressed as [28]:

$$
\phi\left(r^{*}\right) / k_{B} T= \begin{cases}\infty & 0<r^{*}<\sigma \\ \log \left[12 \bar{T} \delta\left(R^{\prime}-\sigma\right) / R^{\prime}\right] & \sigma<r^{*}<R^{\prime} \\ 0 & r^{*}>R^{\prime}\end{cases}
$$

where $\bar{T}$ is the dimensionless temperature of the fluid, $\sigma$ is the diameter of the hard sphere and it is assumed that $R^{\prime} \approx \sigma$ for a single species fluid. $\delta$ is the dirac delta function defining the limit to the infinitely narrow well potential.

Perram and Smith [32] extended the work of Baxter to a fluid mixture. The potential $\phi_{\alpha \beta}$ is then used to define the adsorption capability of the hard spheres. They redefined the infinitely narrow well potential for a fluid mixture:

$$
\phi_{\alpha \beta}(r) / k_{B} T= \begin{cases}\log \left[12 \bar{T}_{\alpha \beta} \delta / R_{\alpha \beta}\right] & r^{*} \leq R_{\alpha \beta} \\ 0 & r^{*}>R_{\alpha \beta}\end{cases}
$$

where $R_{\alpha \beta}=\left(\sigma_{\alpha}+\sigma_{\beta}\right) / 2$.

Assuming $c_{\alpha \beta}^{*}=0$ as $r^{*}>R_{\alpha \beta}$ and using Wiener-Hopf factorisation [41], equation (4) $[31,42]$ becomes:

$$
\begin{aligned}
r^{*} h_{\alpha \beta}^{*}\left(\left|r^{*}\right|\right)= & -\frac{d}{d r^{*}} q_{\alpha \beta}\left(r^{*}\right) \\
& +2 \pi \sum_{\gamma=1}^{M+1} \rho_{\gamma}^{*} \int_{S_{\alpha \gamma}}^{R_{\alpha \gamma}} q_{\alpha \gamma}(s)\left(r^{*}-s\right) h_{\gamma \beta}^{*}\left(\left|r^{*}-s\right|\right) d s
\end{aligned}
$$

where $S_{\alpha \beta}=\left(\sigma_{\alpha}-\sigma_{\beta}\right) / 2$. Perram [43] solved equation (8) using the virial expansion to express the equilibrium pressure as a power series function of density. Perram and Smith [32] showed that after truncating the high order terms in the virial expansion, for $S_{\alpha \beta}<r<R_{\alpha \beta}$, the indirect correlation function becomes: 


$$
h_{\alpha \beta}^{*}\left(r^{*}\right)=-1+\frac{1}{12} \lambda_{\alpha \beta} R_{\alpha \beta} \delta\left(r^{*}-R_{\alpha \beta}\right)
$$

where $\lambda_{\alpha \beta}$ will be determined later.

There are two possible domains:

A) $S_{\alpha \beta}<r^{*}<R_{\alpha \beta}$,

$$
q_{\alpha \beta}\left(r^{*}\right)=a_{\alpha}\left(r^{* 2}-R_{\alpha \beta}^{2}\right) / 2+b_{\alpha}\left(r^{*}-R_{\alpha \beta}\right)+\lambda_{\alpha \beta} R_{\alpha \beta}^{2} / 12
$$

where

$$
\begin{aligned}
& a_{\alpha}=\left(1-\zeta_{3}+3 R_{\alpha} \zeta_{2}\right) /\left(1-\zeta_{3}\right)^{2}-X_{\alpha} /\left(1-\zeta_{3}\right) \\
& b_{\alpha}=\left(-3 R_{\alpha}^{2} \zeta_{2}\right) / 2\left(1-\zeta_{3}\right)^{2}-R_{\alpha} X_{\alpha} / 2\left(1-\zeta_{3}\right) \\
& X_{\alpha}=\frac{\pi}{6} \sum_{\gamma=1}^{M+1} \rho_{\gamma}^{*} \lambda_{\alpha \gamma} R_{\alpha \gamma}^{2} \sigma_{\gamma} \\
& \zeta_{j}=\frac{\pi}{6} \sum_{\gamma=1}^{M+1} \rho_{\gamma}^{*} \sigma_{\gamma}^{j}, \quad j=1,2,3
\end{aligned}
$$

B) $r^{*}>R_{\alpha \beta}$,

$$
q_{\alpha \beta}\left(r^{*}\right)=\frac{d}{d r^{*}} q_{\alpha \beta}\left(r^{*}\right)=0
$$

Assuming the planar or flat surface is itself species $(M+1)$, particle diameter $\sigma_{M+1} \rightarrow \infty$ and the number density or particle density $\rho_{M+1}^{*} \rightarrow 0$, equation (8) can be now rewritten as:

$$
\begin{aligned}
r^{*} h_{\alpha \beta}^{*}\left(\left|r^{*}\right|\right)= & -\frac{d}{d r^{*}} q_{\alpha \beta}\left(r^{*}\right) \\
& +2 \pi \sum_{\gamma=1}^{M} \rho_{\gamma}^{*} \int_{S_{\alpha \gamma}}^{R_{\alpha \gamma}} q_{\alpha \gamma}(s)\left(r^{*}-s\right) h_{\gamma \beta}^{*}\left(\left|r^{*}-s\right|\right) d s
\end{aligned}
$$

The $\lambda_{\alpha \beta}$ parameter can be related to the dimensionless temperature of the system $\bar{T}$ using the approximation proposed by Perram and Smith [42]. The relationship can be written as follow:

$$
\lambda_{\alpha \beta} \bar{T}_{\alpha \beta}=a_{\alpha}+b_{\alpha} / R_{\alpha \beta}+\frac{\pi}{6} \sum_{\gamma=1}^{M+1} \rho_{\gamma} \frac{\lambda_{\beta \gamma} R_{\beta \gamma}^{2}}{R_{\alpha \beta}} q_{\alpha \gamma}\left(S_{\alpha \gamma}\right)
$$


In this study, the hard spheres are considered not to adhere/adsorb to each other but only to the wall $\left(\lambda_{\alpha \beta} \approx 0, \alpha, \beta \neq M+1\right)[34,42,44]$. Hence, this limits spheresphere interaction solely to the repulsive hard core potential. If needed, this condition can be relaxed to account for inter-molecular adsorption. With the wall species diameter, $\sigma_{M+1} \rightarrow \infty$, the adsorption parameter, $d_{\alpha}$ can be defined as [34]:

$$
d_{\alpha}(T)=\int_{0}^{\infty}\left[e^{-\phi_{\alpha}\left(r^{*}\right) / k_{B} T}-1\right] d r^{*}
$$

The corresponding infinitely narrow well potential, $\phi_{\alpha}$ for hard spheres adsorbing to the planar wall in equation (14) is [34]:

$$
\phi_{\alpha}\left(r^{*}\right)= \begin{cases}-\epsilon\left(\sigma_{\alpha}-r^{*}\right) / \sigma_{\alpha} & 0<r^{*}<\sigma_{\alpha} \\ 0 & r^{*}>\sigma_{\alpha}\end{cases}
$$

where $\epsilon$ is the adsorption energy per unit $k_{B} T$. Substituting $a_{\alpha}, b_{\alpha}$ and $S_{\alpha}$ into equation (13), $\lambda_{\alpha, M+1}$ can be determined using the following expression [34]:

$$
\frac{\lambda_{\alpha, M+1} R_{\alpha, M+1}}{12 d_{\alpha}(T)}=\frac{1-\zeta_{3}+3 \sigma_{\alpha} \zeta_{2}}{\left(1-\zeta_{3}\right)^{2}}-\frac{\pi \sigma_{\alpha}}{12\left(1-\zeta_{3}\right)} \sum_{\gamma=1}^{M} \rho_{\gamma}^{*} \sigma_{\gamma} \lambda_{\gamma, M+1} R_{\gamma, M+1}
$$

Therefore, for $r^{*}>R_{\alpha \beta}$ and a multi-component fluid, equation (12) becomes $[42,43]$ :

$$
\begin{aligned}
& h_{\alpha \beta}^{*}\left[R_{\alpha \beta}+(m+1) \Delta\right]= \\
& =\frac{\pi \Delta \sum_{\gamma=1}^{M} \sum_{\eta=0}^{N_{\gamma}} \rho_{\gamma}^{*}\left\{\begin{array}{l}
q_{\alpha \gamma}\left(S_{\alpha \gamma}+\eta \Delta\right) \times \\
\left(R_{\gamma \beta}+(m+1-\eta) \Delta\right) \times \\
h_{\gamma \beta}^{*}\left[\left|R_{\gamma \beta}+(m+1-\eta) \Delta\right|\right]
\end{array}\right\}}{\left(R_{\alpha \beta}+(m+1) \Delta\right)}
\end{aligned}
$$

where $m(=1,2,3 \ldots \ldots)$ is the grid point along hard sphere separation axis, $N_{\gamma}\left(=\sigma_{\gamma} / \Delta\right)$ is the limit to the integral term in equation (8) and $\Delta$ is the grid step size.

The pair correlation function, $g_{\alpha \beta}^{*}$ can be calculated once the indirect correlation function, $h_{\alpha \beta}^{*}$ is obtained from equation (16). The solvation pressure $p_{s}$ generated by hard spheres confined by flat bodies is computed from equation (2), using a forward finite difference scheme [11]:

$$
p_{s}[m \Delta]=-\frac{k_{B} T}{2 \pi R_{e f f} \Delta^{2}}\left\{\begin{array}{l}
g_{M+1, M+1}^{*}[(m+1) \Delta]-2 g_{M+1, M+1}^{*}[m \Delta] \\
+g_{M+1, M+1}^{*}[(m-1) \Delta]
\end{array}\right\}
$$




\subsection{Shear stress}

Friction generated due to the shear of ultra-thin films adsorbed on smooth surfaces is non-Newtonian. Eyring [45] assumed that for closely-packed molecules, the motion of a volume of fluid must overcome a cage-like potential barrier:

$$
E_{y}=Q_{y}+p \Omega_{y}-\tau_{y} \phi_{y}^{*}
$$

where $E_{y}$ is the barrier height, $Q$ is the process activation energy, $\Omega_{y}$ is the pressureinduced activation volume, $p$ is the pressure and $\phi_{y}^{*}$ is the shear-induced activation volume.

It is assumed that the wall-wall pressure is equal with the solvation pressure $\left(p=p_{s}\right)$ given by equation (17). Therefore, the barrier height, $E_{y}$ is equivalent to the solvation energy, $W^{*}$ (see equation (1)) for the hard sphere particles confined by flat bodies. Using a backward finite differencing scheme for equation (1), $E_{y}\left[\mathrm{~J} / \mathrm{m}^{2}\right]$ for hard spheres confined by flat bodies becomes:

$$
E_{y}[m \Delta]=\frac{k_{B} T}{2 \pi R_{e f f} \Delta}\left\{g_{M+1, M+1}^{*}[m \Delta]-g_{M+1, M+1}^{*}[(m-1) \Delta]\right\}
$$

where $m$ is the grid points $(1,2,3 \ldots$.$) and \Delta$ is the grid step size

By rearranging equation (18), the shear stress, $\tau_{y}$ can be defined as:

$$
\tau_{y}=\frac{Q_{y}+p_{s} \Omega_{y}-E_{y}}{\phi_{y}^{*}}
$$

The parameters $Q_{y}, \Omega_{y}$ and $\phi_{y}^{*}$ must be obtained experimentally. Here, the values measured by He et al [46] are considered as a first approximation $\left(Q_{y}=1.33 \times 10^{-20} \mathrm{~J}\right.$, $\Omega_{y}=1.93 \times 10^{-13} \mathrm{~m}^{3} / \mathrm{m}^{2}$ and $\left.\phi_{y}^{*}=1.21 \times 10^{-12} \mathrm{~m}^{3} / \mathrm{m}^{2}\right)$. The shear-induced activation

volume, $\phi_{y}^{*}$ is interpreted as the size of segment that moves during the shear stress process. This volume can be a part of a molecule or a dislocation line. The pressure activation volume, $\Omega_{y}$ is associated with the local increase in volume to permit the molecular motion to occur.

\section{Results and Discussions}

First the case of an idealised mono-molecular fluid intervening between a pair of flat ultra-smooth solid barriers is considered. The bounding solid surfaces are assumed to represent typical smooth asperity peak summits of an opposing pair on semi-infinite surfaces in close contiguity, thus subject to ultra light loads. These conditions promote solvation pressure predicted by equation (17) and shear in relative motion of molecular layers (equation (20)) when the asperity summits undergo mutual approach. This initial case study attempts to understand the inter-molecular interactions including those between the assumed hard spheres with the solid barriers through to their adsorption. 
Then, the study is extended to the case of a simple physical fluid of bi-molecular structure.

The idealised fluid comprises a single species of assumed hard spherical molecules, confined by planar walls (figure 2). The molecular diameter is assumed to be $1 \mathrm{~nm}$. An example of such an idealised fluid is OMCTS.

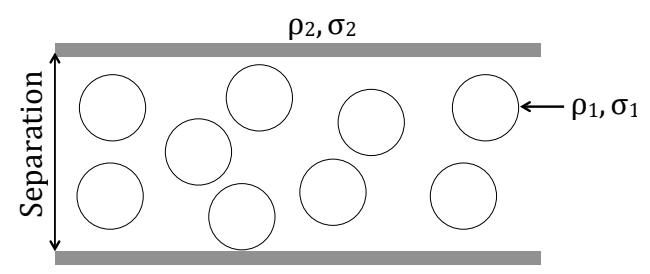

Figure 2. Single component fluid confined by planar walls

The pair correlation function between the hard spheres, $g_{11}^{*}$ can be obtained as shown in figures 3 (a) and (b) through solution of the OZ-PY equation. The packing fraction, $\eta_{\alpha}^{*}$ denotes the occupied volume fraction by the molecular disposition and can be expressed as:

$$
\eta_{\alpha}^{*}=\pi \rho_{\alpha}^{*} \sigma_{\alpha}^{3} / 6
$$

A smaller packing fraction, $\eta_{1}^{*}$ represents a smaller number density, $\rho_{1}^{*}$. Thus, the amplitude of oscillations reduces significantly for a smaller packing fraction, $\eta_{1}^{*}$, attenuating the effect of solvation. It can be observed that $g_{11}^{*}$ behaves in an oscillatory manner as the separation gap is reduced. However, due to the increased packing fraction, the interaction between particles $g_{11}^{*}$ for $\eta_{1}^{*}=0.4$ promotes a larger amplitude.

Figure 3 also shows the pair correlation function for hard sphere-wall $g_{21}^{*}$ and wallwall $g_{22}^{*}$ interactions. It is noted that the $g_{21}^{*}$ is negative at small separations which reflects a strong exclusion of the hard spheres from the confined region (figure $3(\mathrm{c})$ and (d)). It can be observed that with increasing adsorption energy, $\epsilon_{1}$, a sharp peak becomes apparent at the separation height of $1 \mathrm{~nm}$ (the molecular diameter of the species). This peak is caused by the packing of the hard spheres onto the already adsorbed mono-layer residing on the planar wall surface. The increment $\epsilon_{1}$ encourages adsorption of hard spheres to form a denser layer on the planar wall. The adsorption of an increasing number of hard spheres, packing on an already formed monolayer instead of the bare planar wall reduces the oscillatory behaviour there. This is true for both $g_{21}^{*}$ and $g_{22}^{*}$. For smaller values of $\eta_{1}^{*}$ (figures 3 (a) and (c)), the packing of the hard spheres onto the monolayer at the planar surface resembles the observations made by Perram and Smith $[42]$. 


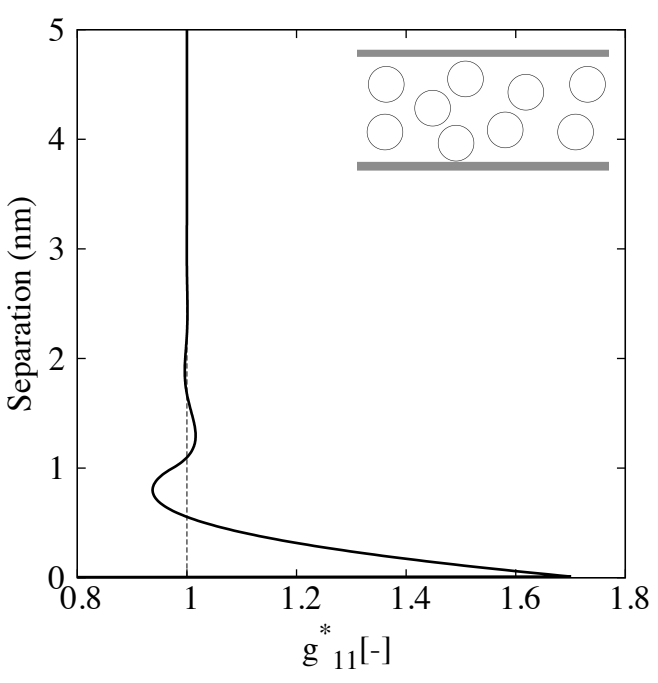

(a) $\eta_{1}^{*}=0.2$

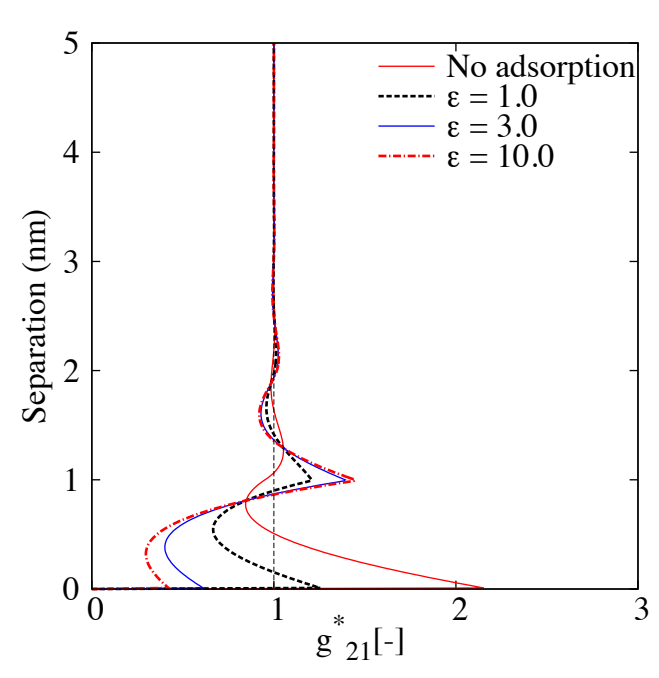

(c) $\eta_{1}^{*}=0.2$

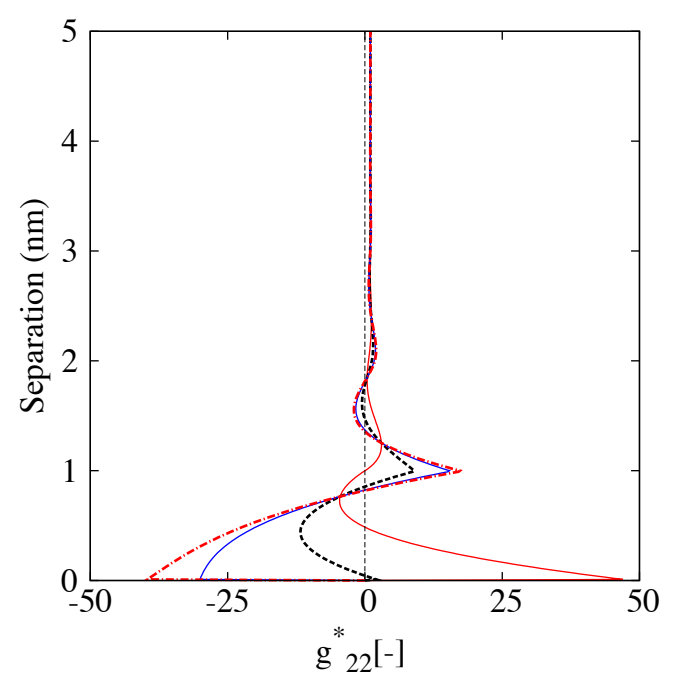

(e) $\eta_{1}^{*}=0.2$

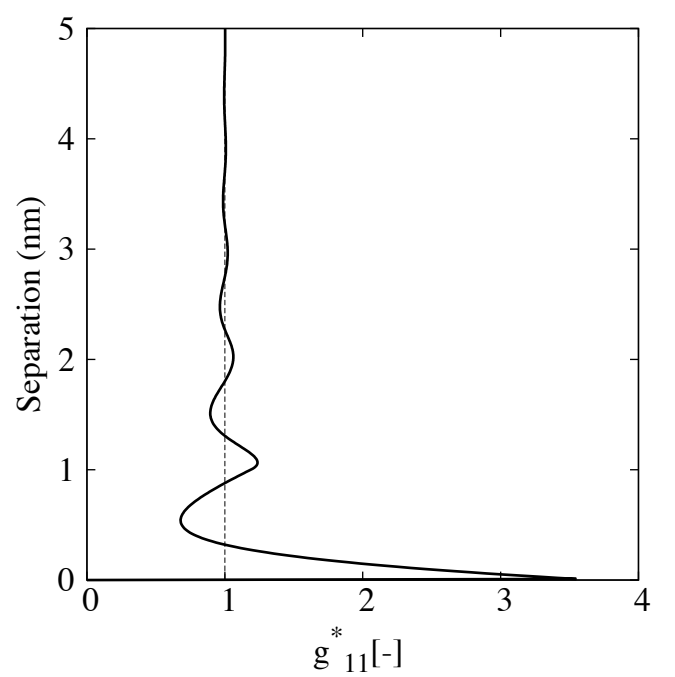

(b) $\eta_{1}^{*}=0.4$

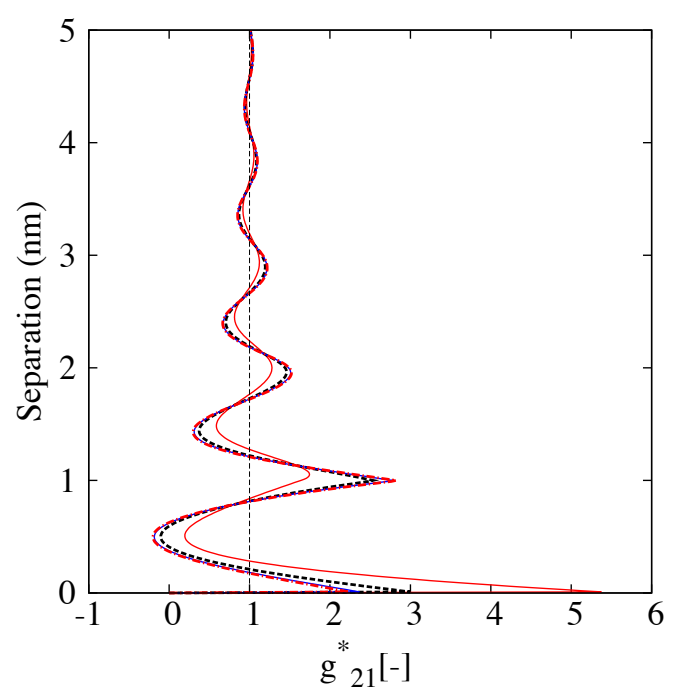

(d) $\eta_{1}^{*}=0.4$

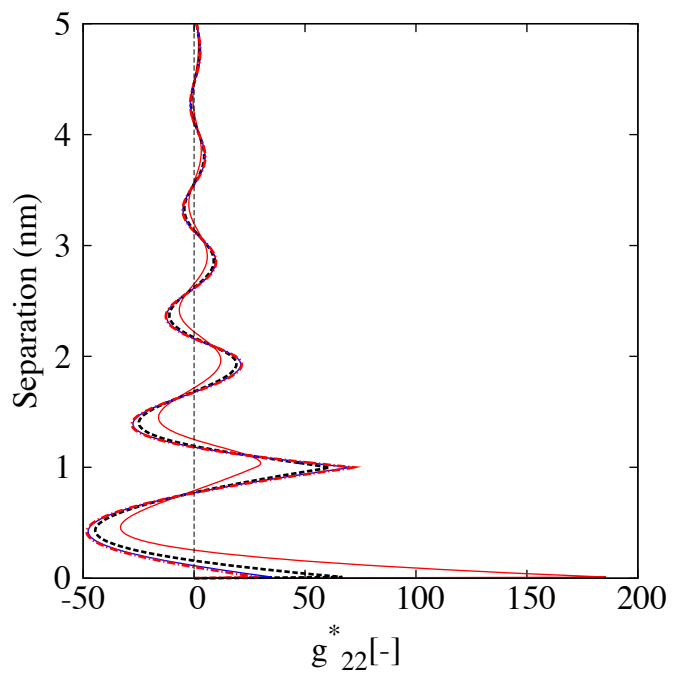

(f) $\eta_{1}^{*}=0.4$

Figure 3. Pair correlation functions, $g^{*}$ for a single component fluid with varying adsorption energy, $\epsilon$ for two different packing fractions, $\eta_{1}^{*}$ 
Figure 4 illustrates the solvation pressure computed using different adsorption energy values, $\epsilon_{1}$. Higher values of $\epsilon_{1}$ encourage the formation of a denser monolayer. Hence, this increases the solvation pressure at separations near the monolayer. A smaller $\eta_{1}^{*}$ value (see figure 4 (a)) reduces the solvation pressure due to the dilution of the fluid. An interesting point is to note that solvation pressure is noticeable only beyond $2 \mathrm{~nm}$. This is because the separation of the walls is limited by the adsorbed monolayers on the contiguous approaching walls.

The packing fraction, $\eta_{1}^{*}=0.4$ is used by Henderson and Lozada-Cassou [17] and Matsuoka and Kato [11] to compute the solvation pressure for OMCTS. Therefore, a comparison can be made between the solvation pressure computed based on this packing fraction and that obtained by Chan and Horn empirically (see figure 4 (b)) for OMCTS [9]. It can be observed that both methods show good agreement in terms of oscillatory characteristics but at a slightly varying amplitudes.

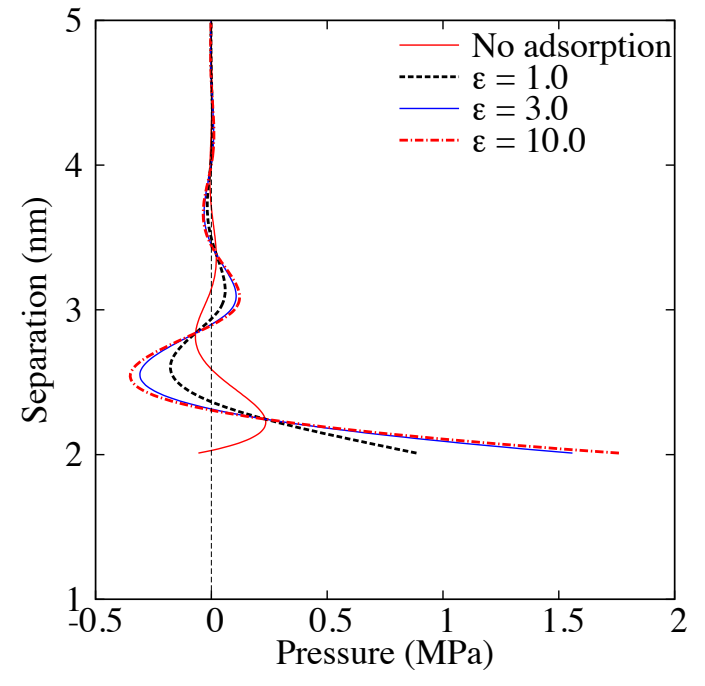

(a) $\eta_{1}^{*}=0.2$

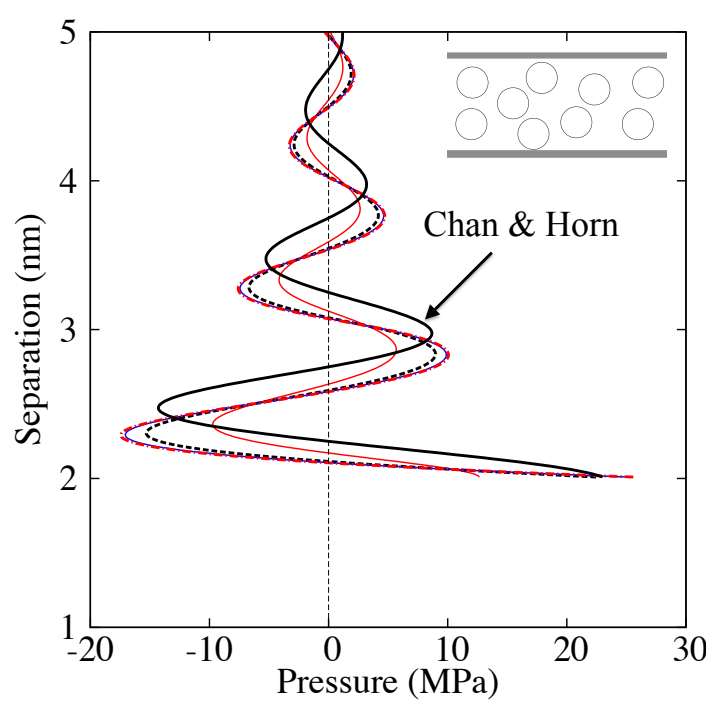

(b) $\eta_{1}^{*}=0.4$ (Comparison with Chan and Horn's [9] approach)

Figure 4. Solvation pressure for a single component fluid confined by planar walls with varying adsorption energy, $\epsilon$ for two different packing fractions, $\eta_{1}^{*}$

The solvation energy required to compute the shear stress using the Eyring model [45] is plotted in figure 5 for different adsorption energy levels of hard spheres. The energy has a larger amplitude for $\eta_{1}^{*}=0.4$ due to increased molecular solvation. Using equation (20), the shear stress can be obtained for a wall-wall $\left(g_{22}^{*}\right)$ interaction as shown in figure 6. Negative shear stress in the figure is discussed later. 


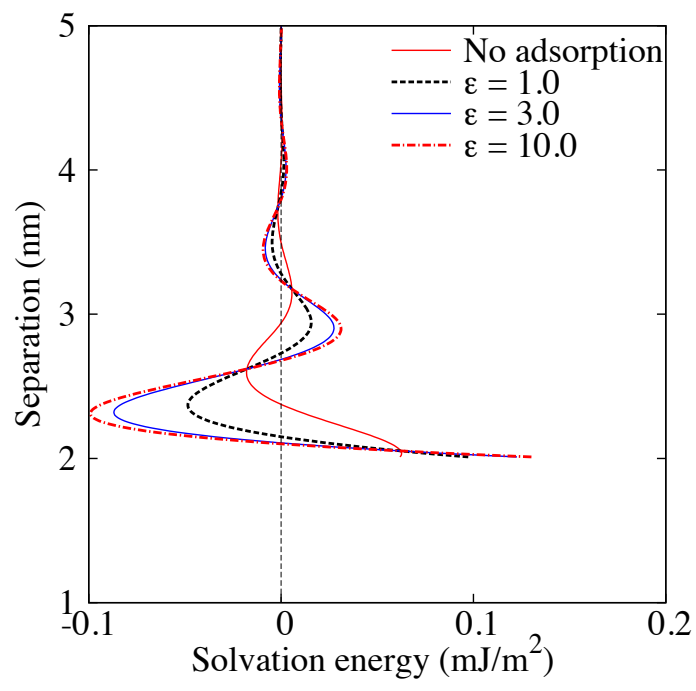

(a) $\eta_{1}^{*}=0.2$

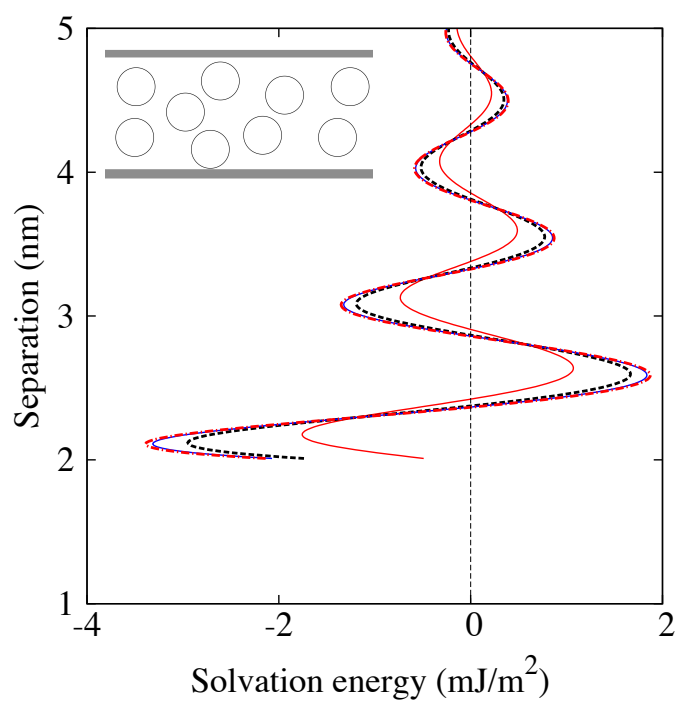

(b) $\eta_{1}^{*}=0.4$

Figure 5. Solvation energy for a single component fluid confined by planar walls with varying adsorption energy, $\epsilon$ for two different packing fractions, $\eta_{1}^{*}$

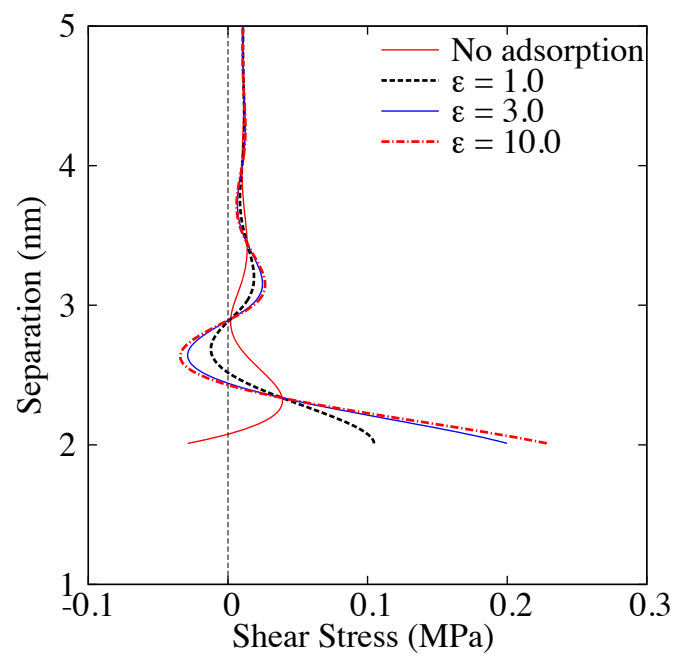

(a) $\eta_{1}^{*}=0.2$

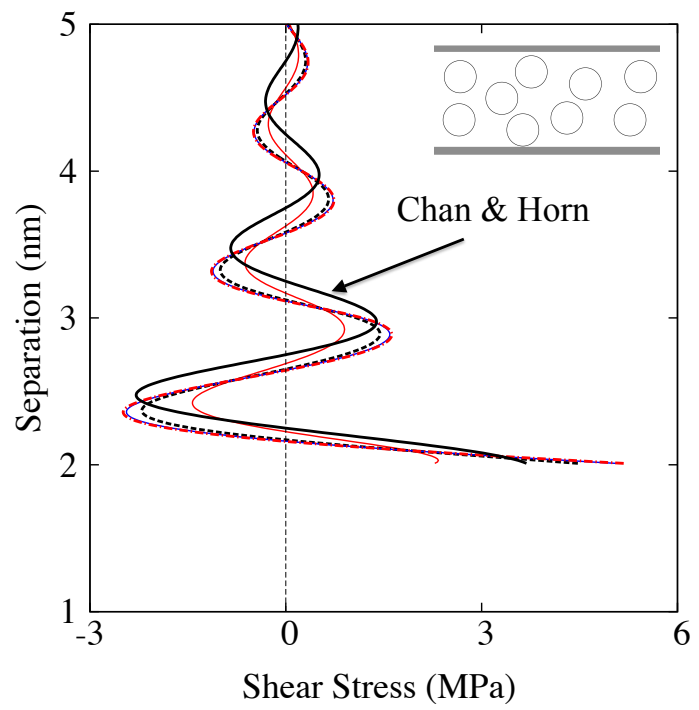

(b) $\eta_{1}^{*}=0.4$ (Comparison with Chan and Horn's [9] approach)

Figure 6. Shear stress for a single component fluid confined by planar walls with varying adsorption energy, $\epsilon$ for two different packing fractions, $\eta_{1}^{*}$

Real physical fluids comprise a multitude of molecular species. The model described thus far can be extended to a simple physical fluid containing two molecular components (figure 7). The empirical method derived by Chan and Horn [9] is only limited to a single component fluid system. The same limitation does not apply to the model developed here. In the model of figure 7, the bi-molecular species are represented by hard spheres, 
one of nominal average diameter $0.5 \mathrm{~nm}$ (species 1) and the other of nominal average diameter $1 \mathrm{~nm}$ (species 2). It is assumed that the species 1 has significantly higher adsorption energy than species 2 . This choice is purely arbitrary.

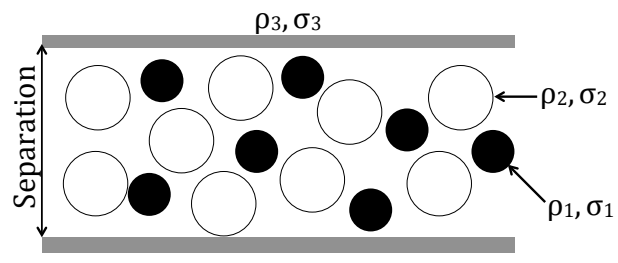

Figure 7. Dual component fluid confined by planar walls

The overall packing fraction for the fluid mixture is assumed to remain constant at $\eta_{t o t}^{*}=0.4$. Therefore, for a given concentration ratio, $r_{w}$ of the species, their packing fractions are obtained as:

$$
\eta_{1}^{*}=r_{w} \eta_{t o t}^{*}, \quad \eta_{2}^{*}=\left(1-r_{w}\right) \eta_{t o t}^{*}
$$

Figure 8 shows pressure perturbations and discontinuous shear stress variations caused by the local minima in the intermolecular pair correlation functions (local potentials between individual molecules of the same or different species). The effect of molecular adsorption energy on the solid barrier, as well as the molecular concentrations are investigated. There is an ideal concentration of each component, which leads to the optimal (minimum or maximum) load carrying capacity and shear. The figure shows that for a higher molecular packing fraction, both the solvation pressure and shear stress levels increase. The increase in pressure is due to a higher density of the molecules in the fluidic medium. Therefore, the density fluctuation near the solid barriers due to solvation effect would be higher. Similarly, a higher shear stress would be expected with the greater packing fraction because friction caused by the layering effect (i.e. ejection of rows of molecules out of the conjunction) would be subjected to the presence of a larger number of molecules. The lower packing fraction would be representative of a medium tending to a vapour or gaseous environment. On the other hand, the higher packing faction of $\eta_{t o t}^{*}=0.4$ is more representative of a liquid medium. Therefore, the conclusions arrived at with regard to figure 8 are only natural. Figure 8 also shows results for different concentrations of the species with greater adsorption energy. 


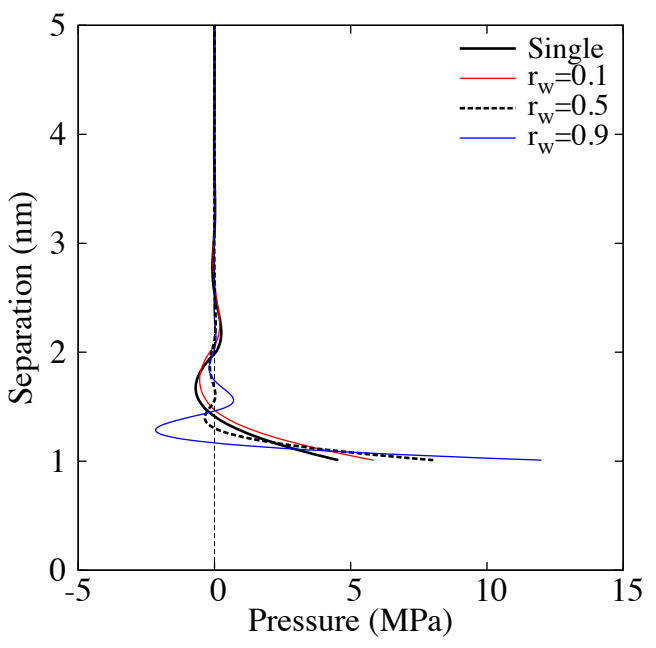

(a) $\eta_{\text {tot }}^{*}=0.2$

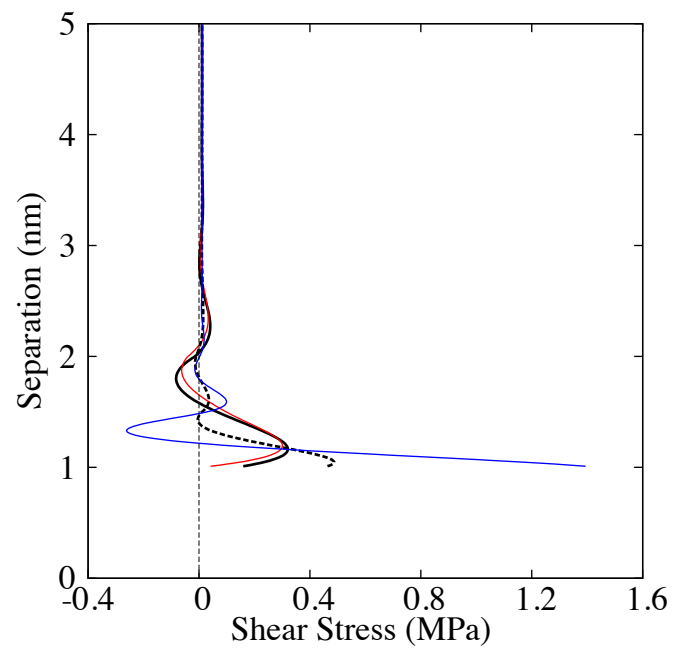

(c) $\eta_{\text {tot }}^{*}=0.2$

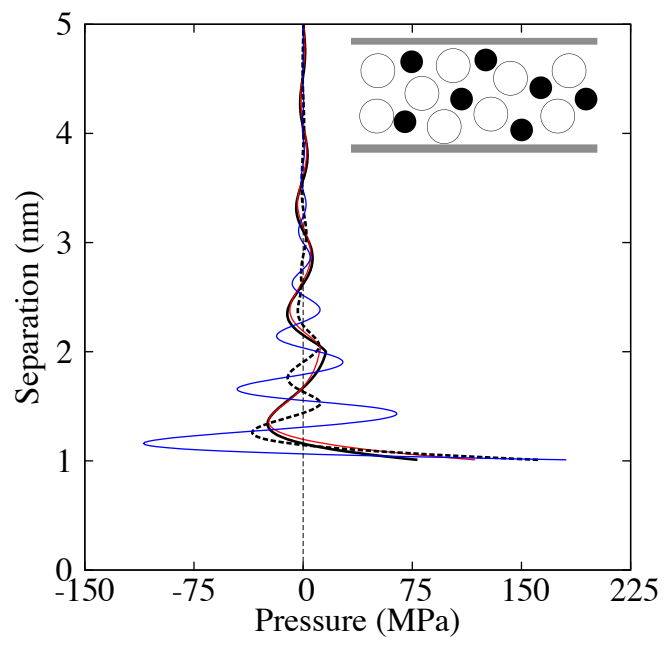

(b) $\eta_{\text {tot }}^{*}=0.4$

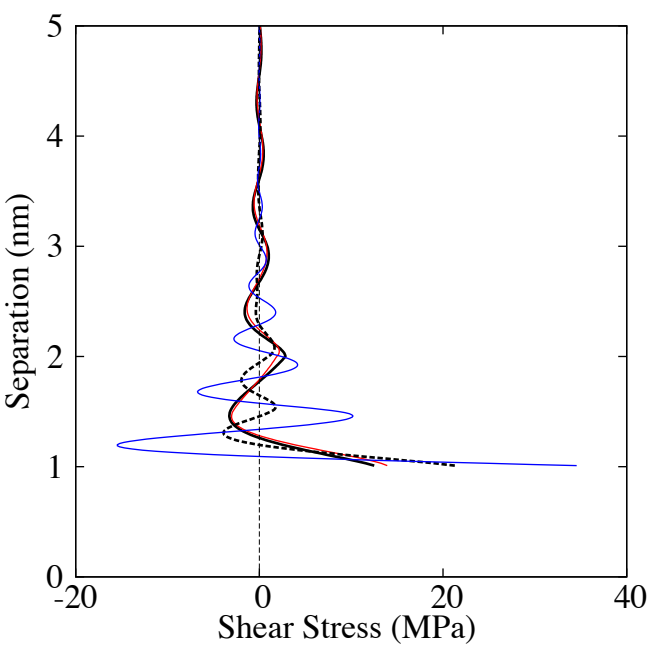

(d) $\eta_{\text {tot }}^{*}=0.4$

Figure 8. Pressure and shear stress for a dual component fluid system confined by planar walls with varying concentration ratio, $r_{w}$ for species 1 for different packing fractions, $\eta_{t o t}^{*}$ (Assuming adsorption energy for species 1 and $2: \epsilon_{1}=10, \epsilon_{2}=0.1$ )

Differences are noted between generated pressures and shear with different concentration levels. This is more evident in figure 9, where for a given packing fraction, larger concentrations of the species with higher adsorption energy lead to formation of a thicker adsorbed layer. This tends to create a lower shear strength film, thus reducing shear. This is inline with the observation of Jahanmir and Beltzer [37]. However, it is clear in figures 9 (c) and (d) that after a minimum shear stress level is achieved, any further accumulation of adsorbed molecules increases the shear stress. It can surmised that thicker adsorbed films result in an effective increase in viscosity of the film, thus a greater shear stress results. This maybe the underlying explanation for the observed micro-scale phenomena of shear stress variation in direct proportion to fluid viscosity. 
Figures 9 (a) and (b) show that for any packing fraction, generated pressures increase with the greater concentration of the species with higher adsorption energy. With sufficient concentration, a plateau effect is observed which infers that the adsorbed layer inhibits solvation to an extend. However, as a further increase in concentration takes place, the density variation near the surfaces reaches a saturation point, where the solvation effect seems to exacerbate again. With the solvation phenomenon, rows of molecules are ejected at discrete energy levels as shown by Chong et al [27]. It is, therefore, natural that with an increasing concentration, there would be a limit where the advantages gained through reduced shear strength of a layer is lost by the energy required to eject a larger number of molecules out of a conjunction of a mutually approaching solid barriers. Referring to the micro-scale behaviour of fluidic films in conjunctions subject to pure squeeze, shear stress is governed by $\tau= \pm(h / 2)\left(\partial p_{s} / \partial x\right)$, where $x$ is the direction normal to the line of mutual approach. The pressure gradient here is a function of number of layers and molecules in each layer which are subject to solvation pressure (discretisation and ejection of rows of molecules). Therefore, the larger number of molecules and thicker adsorbed films constitute a higher shear stress. In an allusion to the micro-scale behaviour, the underlined nano-scale phenomena observed here indicates that changes in oscillatory behaviour of solvation pressure (attraction-repulsion) alters the shearing characteristics of surface adsorbed films. Since the changes in pressure gradient results in layering of the adsorbed film, the layers of molecules rearrange, thus alter the sense of shear. Negative shear occurs in transition between discrete layers of the adsorbed film. This explains the negative shear stress noted in figure 6 . 

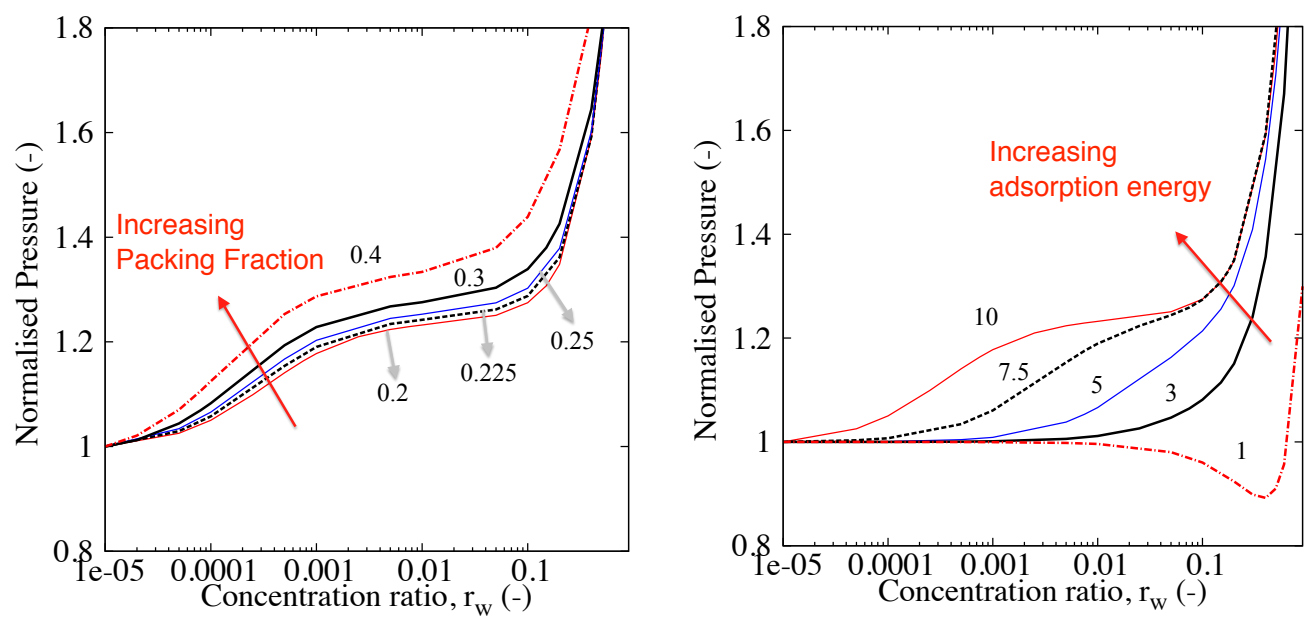

(a) Packing fraction, $\eta_{\text {tot }}^{*}$ variation $\left(\epsilon_{1}=(\mathrm{b})\right.$ Adsorption energy, $\epsilon_{1}$ variation $\left(\eta_{\text {tot }}^{*}=\right.$ $\left.10, \epsilon_{2}=0.1\right)$ $\left.2, \epsilon_{2}=0.1\right)$
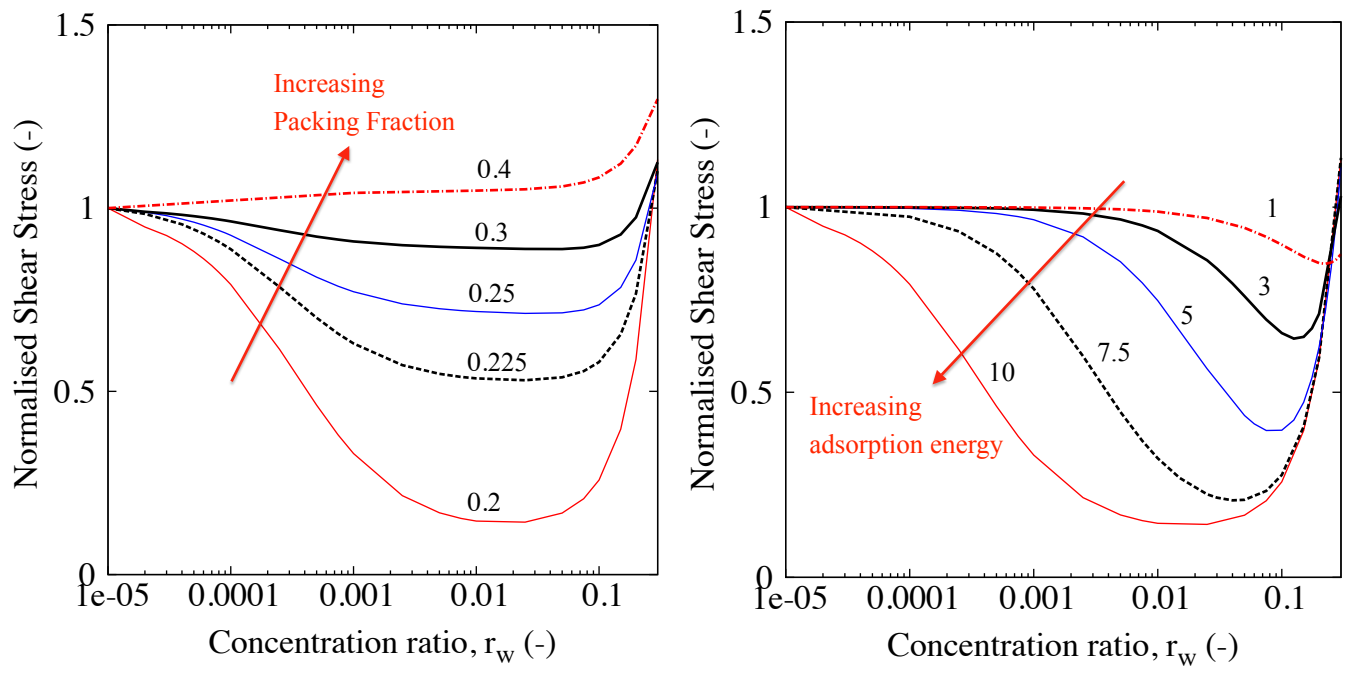

(c) Packing fraction, $\eta_{\text {tot }}^{*}$ variation $\left(\epsilon_{1}=(\mathrm{d})\right.$ Adsorption energy, $\epsilon_{1}$ variation $\left(\eta_{\text {tot }}^{*}=\right.$ $\left.10, \epsilon_{2}=0.1\right)$

$\left.2, \epsilon_{2}=0.1\right)$

Figure 9. Normalised pressure and shear stress at $1 \mathrm{~nm}$ for a dual component fluid system considering different concentration ratio, $r_{w}$ for species 1

\section{Conclusion}

When a simple physical fluid is considered with a mix of molecular species, some with boundary active characteristics, a surface adsorbed film of low shear strength can result. At nano-scale the shear characteristics are dependent on the adsorption energy of the molecular species, their packing fraction and their concentration within the fluidic medium. In the presence of smooth solid barrier surfaces, the oscillatory solvation effect is inhibited by formation of these adsorbed films with reduced shear strength. However, further accumulation of the adsorbed molecules reintroduces solvation by increased density of molecules competing to adhere to the already adsorbed film on the boundary solids. The conclusion of this work is that there exists an optimum packing 
order and molecular mix and disposition for any coherent boundary active film.

\section{Acknowledgement}

The authors acknowledge the technical support from partners and sponsorship provided by the EPSRC through the ENCYCLOPAEDIC program grant.

\section{References}

[1] A. Erdemir. Review of engineered tribological interfaces for improved boundary lubrication. Tribology International, 38(3):249-256, 2005.

[2] A. Einstein and L. Infeld. The evolution of physics: the growth of ideas from early concepts to relativity and quanta. Simon and Schuster, New York, 1938.

[3] A. Mulero (Ed.). Theory and Simulation of Hard-Sphere Fluids and Related Systems. Springer, Berlin, 2008.

[4] E. M. Lifshitz. The theory of molecular attractive forces between solids. Soviet Physics JETP, 2:94-110, 1956.

[5] R. Gohar and H. Rahnejat. Fundamentals of tribology. Imperial College Press, London, 2008.

[6] J. N. Israelachvili. Intermolecular and surface forces. Academic press London, 1992.

[7] P. Attard and J. L. Parker. Oscillatory solvation forces: A comparison of theory and experiment. Journal of Physical Chemistry, 96(12):5086-5093, 1992.

[8] R. G. Horn and J. N. Israelachvili. Direct measurement of structural forces between two surfaces in a nonpolar liquid. Journal of Chemical Physics, 75(3):1400-1412, 1981.

[9] D. Y. C. Chan and R. G. Horn. The drainage of thin liquid films between solid surfaces. Journal of Chemical Physics, 83(10):5311-5324, 1985.

[10] R. Y. H. Lim and S. J. O'Shea. Discrete solvation layering in confined binary liquids. Langmuir, 20(12):4916-4919, 2004.

[11] H. Matsuoka and T. Kato. An ultrathin liquid film lubrication theory - calculation method of solvation pressure and its application to the ehl problem. Journal of Tribology, 119:217-226, 1997.

[12] M. Al-Samieh and H. Rahnejat. Ultra-thin lubricating films under transient conditions. Journal of Physics D: Applied Physics, 34:2610-2621, 2001.

[13] M. F. Abd. Al-Samieh and H. Rahnejat. Nano-lubricant film formation due to combined elastohydrodynamics and surface force action under isothermal conditions. Proceedings of Institute of Mechanical Engineers, Part C : Journal of Mechanical Engineering Science, 215:1019-1029, 2001.

[14] M. Al-Samieh and H. Rahnejat. Physics of lubricated impact of a sphere in a plate in a narrow continuum to gaps of molecular dimensions. Journal of Physics D: Applied Physics, 35:23112326, 2002.

[15] S. Jang and J. A. Tichy. Rheological models for thin film ehl contacts. Journal of Tribology, 117:22-28, 1995.

[16] D. J. Mitchell, B. W. Ninham, and B. A. Pailthorpe. Hard sphere structural effects in colloid systems. Chemical Physics Letters, 51(2):257-260, 1977.

[17] D. Henderson and M. Lozada-Cassou. A simple theory for the force between spheres immersed in a fluid. Journal of Colloid and Interface Science, 114(1):180-183, 1986.

[18] J. K. Percus and G. J. Yevick. Analysis of classical statistical mechanics by means of collective coordinates. Physical Review, 110:1-13, 1958.

[19] L. Snook and W. van Megen. Structure of dense liquids at solid interfaces. Journal of Chemical Physics, 70:3099-3105, 1979. 
[20] P. Tarazona and L. Vicente. A model for density oscillations in liquids between solid walls. Molecular Physics, 56:557-572, 1985.

[21] S. A. Somers, A. V. McCormick, and H. T. Davis. Superselectivity and solvation forces of a two component fluid adsorbed in slit micropores. Journal of Chemical Physics, 99:9890, 1993.

[22] S. Karanikas, J. Dzubiella, A. Moncho-Jord, and A. A. Louis. Density profiles and solvation forces for a yukawa fluid in a slit pore. Journal of Chemical Physics, 128:204704, 2008.

[23] M. Teodorescu, S. Balakrishnan, and H. Rahnejat. Physics of ultra-thin surface films on molecularly smooth surfaces. Proceedings of the Institution of Mechanical Engineers, Part $N$ : Journal of Nanoengineering and Nanosystems, 220(1):7-19, 2006.

[24] K. N. G. Fuller and D. Tabor. The effect of surface roughness on the adhesion of elastic solids. Proceedings of the Royal Society of London.Series A, Mathematical and Physical Sciences, pages 327-342, 1975.

[25] K. L. Johnson and I. Sridhar. Adhesion between a spherical indenter and an elastic solid with a compliant elastic coating. Journal of Physics D : Applied Physics, 34:683-689, 2001.

[26] H. Rahnejat, P. M. Johns-Rahnejat, M. Teodorescu, V. Votsios, and M. Kushwaha. A review of some tribo-dynamics phenomena from micro- to nano-scale conjunctions. Tribology International, 42(11-12):1531-1541, 2009.

[27] W. W. F. Chong, M. Teodorescu, and H. Rahnejat. Effect of lubricant molecular rheology on formation and shear of ultra-thin surface films. Journal of Physics D : Applied Physics, 44(16):165302, 2011.

[28] R. J. Baxter. Percus-yevick equation for hard spheres with surface adhesion. Journal of Chemical Physics, 49(6):2770-2774, 1968.

[29] B. V. Derjaguin. Adhesion of small particles. Kolloid-Zeit., 69:155-164, 1935.

[30] L. S. Ornstein and F. Zernike. Accidental deviations of density and opalescence at the critical point of a single substance. Proceedings of the Royal Academy, Amsterdam, 17:793-806, 1914.

[31] R. J. Baxter. Ornstein-zernike relation and percus-yevick approximation for fluid mixtures. Journal of Chemical Physics, 52(9):4559-4562, 1970.

[32] J. W. Perram and E. R. Smith. A model for the examination of phase behaviour in multicomponent systems. Chemical Physics Letter, 35(1):138-140, 1975.

[33] F. Hirata. Molecular Theory of Solvation. Kluwer Academic Publishers, Dordrecht, 2003.

[34] J. W. Perram and E. R. Smith. Percus-yevick theory of adsorption. Chemical Physics Letter, 39(2):328-332, 1976.

[35] P. Studt. Boundary lubrication : adsorption of oil additives on steel and ceramic surfaces and its influence on friction and wear. Tribology International, 22(2):111-119, 1989.

[36] M. Ruths, H. Ohtani, M. L. Greenfield, and S. Granick. Exploring the "friction modifier" phenomenon : nanorheology of n-alkane chains with polar terminus dissolved in n-alkane solvent. Tribology Letters, 6:207-214, 1999.

[37] S. Jahanmir and M. Beltzer. An adsorption model for friction in boundary lubrication. Tribology Transactions, 29(3):423-430, 1985.

[38] M. I Temkin. Adsorption equilibrium and the kinetics of processes on nonhomogeneous surfaces and in the interaction between adsorbed molecules. Journal of Physical Chemistry (USSR), 15:296-332, 1941.

[39] E. Dickinson. Adsorption of sticky hard spheres : Relevance to protein competitive adsorption. Journal of Chemical Society - Faraday Transactions, 88(24):3561-3565, 1992.

[40] J. P. Hansen and I. R. McDonald. Theory of Simple Liquids. Academic press London, 2006.

[41] M. S. Wertheim. Analytic solution of the percus-yevick equation. Journal of Mathematical Physics, 5:643-651, 1964.

[42] J. W. Perram and E. R. Smith. Competitive adsorption via percus-yevick approximation. Proceedings of Royal Society A, 353:193-220, 1977.

[43] J. W. Perram. Hard sphere correlation functions in the percus-yevick approximation. Molecular Physics, 30(5):1505-1509, 1975. 
[44] P. T. Cummings, J. W. Perram, and E. R. Smith. Percus-yevick theory of correlation functions and nucleation effects in the sticky hard-sphere model. Molecular Physics, 31(2):535-548, 1976.

[45] H. Eyring. Viscosity, plasticity, and diffusion as examples of absolute reaction rates. The Journal of chemical physics, 4:283, 1936.

[46] M. He, A. Szuchmacher Blum, G. Overney, and R. M. Overney. Effect of interfacial liquid structuring on the coherence length in nanolubrication. Physical Review Letters, 88(15):154302, 2002 .

\section{Appendix: Nomenclature}

$E_{y} \quad$ Barrier height for Eyring model $(J)$

$M \quad$ Total number of particle species (-)

$N_{\gamma} \quad$ Limit to the integral of Ornstein-Zernike (OZ) equation (-)

$Q_{y} \quad$ Process activation energy $(J)$

$R_{\text {eff }} \quad$ Effective particle diameter $(m)$

$R_{\alpha \beta} \quad$ Reduced particle diameter, $\left(\sigma_{\alpha}+\sigma_{\beta}\right) / 2,(m)$

$S_{\alpha \beta} \quad$ Average diameter difference between interacting particles, $\left(\sigma_{\alpha}-\sigma_{\beta}\right) / 2,(m)$

$T \quad$ Temperature of the fluid system $\left({ }^{\circ} \mathrm{C}\right)$

$\bar{T} \quad$ Dimensionless temperature of the fluid system $(-)$

$W^{*} \quad$ Derjaguin approximation for interaction potential between planar walls $\left(\mathrm{J} / \mathrm{m}^{2}\right)$

$c^{*} \quad$ Indirect correlation function (-)

$d_{\alpha} \quad$ Adsorption parameter for particle species $\alpha(-)$

$g^{*} \quad$ Pair correlation function $(-)$

$h^{*} \quad$ Direct correlation function $(-)$

$k_{B} \quad$ Boltzmann constant $\left(m^{2} \mathrm{kgs}^{-2} \mathrm{~K}^{-1}\right)$

$m \quad$ Grid point $(-)$

$n \quad$ Iteration counter $(-)$

$p \quad$ Pressure $(\mathrm{Pa})$

$p_{s} \quad$ Solvation pressure $(\mathrm{Pa})$

$q_{\alpha \beta} \quad$ Parameter for transformed Ornstein-Zernike (OZ) equation (-)

$r^{*} \quad$ Distance between the centre of two particles $(m)$

$s \quad$ Distance between the centre of a particle towards a reference particle $(m)$

$t \quad$ Time step $(s)$

$\Delta \quad$ Step size (-)

$\Omega_{y} \quad$ Lubricant volume where pressure, $p$ is acting on $\left(m^{3}\right)$

$\alpha, \beta, \gamma$ Particle species (-)

$\delta \quad$ Dirac delta function $(-)$

$\epsilon \quad$ Adsorption energy (-)

$\eta^{*} \quad$ Particle packing fraction $(-)$

$\lambda_{\alpha \beta} \quad$ Adsorption-temperature relation in the "sticky" hard sphere model (-)

$\phi_{\alpha \beta} \quad$ Interaction potential between particles (-)

$\phi_{y}^{*} \quad$ Activation volume $\left(m^{3}\right)$

$\rho^{*} \quad$ Density number of a particle $(-)$ 
$\sigma \quad$ Particle diameter $(m)$

$\tau_{y} \quad$ Shear stress $(\mathrm{Pa})$

$\zeta_{i} \quad$ Parameter for variable $q_{\alpha \beta}$ where $i=1,2$, and $3(-)$ 